\title{
OPTICAL PROCESSES IN QUANTUM WELLS
}

\author{
L.J. SHAM \\ Department of Physics, University of California, San Diego \\ La Jolla, California 92093-0319, USA
}

\begin{abstract}
A review is given of the theoretical framework of exciton dynamics in quantum wells including the spin degrees of freedom. A study is made of various momentum, energy, and spin relaxation mechanisms including the effects of exciton-phonon interaction, the single-particle spin-flips by means of spin-orbit interaction and the exciton spin-flip by means of the exchange interaction. All these competing mechanisms are taken into account in a set of equations governing the time evolution of the exciton spin populations. Solutions are then used to interpret observed time-resolved observations of polarized luminescence spectra. For excitons in a two-dimensional system such as a semiconductor quantum well, the breaking of the translational symmetry in the direction normal to the interface plane has been sliown theoretically by Hanamura, by Andreani and Bassani, and by Citrin to result in a recombination rate much faster than in a three-dimensional system. Yet, experiments show comparable decay rates in two- and three-dimensional excitons. Recent experiments with high time resolutions show two decay times for the total luminescence intensity. The slower one agrees with the one usually observed and interpreted as the radiative recombination time. We shall give an explanation for the fast decay as a combination of radiative recombination and single-particle spin-flip and for the slow decay as the radiative recombination slowed down by the presence of lower energy dark states for excitons with parallel spins. The ability to use the same theory to account for the polarization behavior confirms the importance of the exciton spin dynamics. Furthermore, the longitudinal electric field dependence is used to check our theory of exchange.
\end{abstract}

PACS numbers: 73.20.Dx, 78,55.Cr

\section{Introduction}

Electrons confined in semiconductor heterostructures [1] have been much studied both theoretically and experimentally. From the theory viewpoint, optical processes provide an excellent source for gaining knowledge of the electron dynamics as well as an excellent testing ground of our understanding. From the experiment viewpoint, recent rapid progress in the time-resolved spectroscopic 
measurements in semiconductor heterostructures makes possible very direct measurements of the time evolution of certain electronic processes [2-7]. Yet, interpretation of the measurements requires a good theoretical framework. We propose here to bring out a number of recent issues in optical processes which emphasize the distinct quasi-two-dimensional nature of the quantum wells and which require the interplay of experiment and theory to build an understanding of the underlying electronic dynamics.

In the following, we begin with the basic model of the semiconductor quantum well and the rules governing the optical excitations. We examine a number of features which distinguish the quantum wells from the bulk which make the wells worth studying. Then we describe the physics underlying the dynamics of the excitons including the spin degrees of freedom. Application is made to analyze the measured time-resolved polarization spectra. Finally, we point out a few directions for further research.

\section{Fundamentals of the carriers and the optical processes}

We use the multi-band effective-mass approximation to represent the electron and hole states as products of the envelope functions modulating the Bloch waves at the band edges. In a bulk III-V direct-gap semiconductor, the conduction band edge has $\Gamma_{6}$ symmetry and the $z$-component of the electron's angular momentum is represented by $s$ with $s= \pm \frac{1}{2}$. The valence band edges are the resultant of the spin-orbit mixing of the $p$-like orbitals and spin $1 / 2$. The fourfold degenerate states, called the heavy hole states, have symmetry $\Gamma_{8}$ and the index $m_{\mathrm{v}}$ which describes the valence electron spin states and which is isomorphous to the $z$-component of the angular momentum of spin $\frac{3}{2}$. We shall represent the valence-hole spin states by the time-reversed states of the electronic Bloch states and associate the index $m_{\mathrm{h}}$ with the hole spins so that $m_{\mathrm{h}}=-m_{\mathrm{v}}$. Hole subband mixing from the off-diagonal part of the Luttinger Hamiltonian [8] can be thought of as $k \cdot p$ mixing. Quantum well confinement gives the envelope as free waves along the interface plane with a well-defined wave vector $k_{\|}$and discrete quantum states for motion along the growth axis (normal to the interface plane).

As an example of the optical processes, let us study the photoluminescence, which may be regarded as a three-step process (Fig. 1a):

1. excitation in which a photon is absorbed raising an electron from a valence subband to a conduction subband;

2. relaxation in which the electron in the conduction subband and the hole in the valence subband relax to quasi-equilibrium states near the respective band edges;

3. recombination in which the electron-hole pair annihilates emitting a photon.

In a pump-and-probe experiment, the excitation of the system by intense light replaces step (1) and the absorption of light from a weak probe replaces step (3).

Optical transitions across the direct gap of a III-V semiconductor depend not only on the energy difference of the conduction and valence subband states 


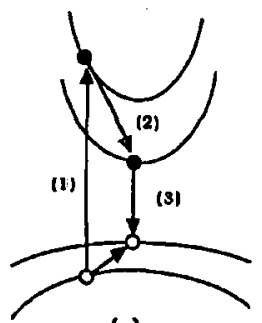

(a)

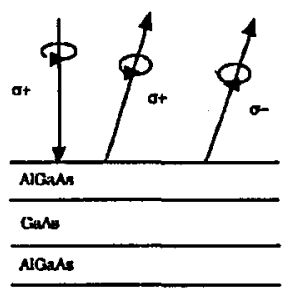

(b)

Fig. 1. (a) Three steps in the optical processes of luminescence across the band gap. (b) An arrangement for luminescence polarization measurement.

but also on the nature of their wave functions. We classify the light with circular polarization $\sigma_{-}$and $\sigma_{+}$respectively with polarization vectors

$$
A_{ \pm}=A_{x}-\mathrm{i} A_{y} \text {. }
$$

We assign photon angular momentum $m_{\mathrm{p}}$ values 1 for the $\sigma_{+}$polarization, -1 for the $\sigma_{-}$polarization, and 0 for the $p$-polarization with vector $A_{z}$. Then a straightforward evaluation of the matrix element of $p-A$ yields the selection rule [9] which expresses the angular momentum conservation along the $z$-axis:

$$
s+m_{\mathrm{h}}=m_{\mathrm{p}} \text {. }
$$

The schematic arrangement shown in Fig. 1b shows a typical way to measure the luminescence polarization. A circularly polarized light, say $\sigma_{+}$, is normally incident on the quantum well. The emitted light at a small angle to the normal is passed through a polarizer and the intensity of the light for each polarization $\sigma_{+}$and $\sigma_{-}$is measured. The fractional difference of the intensities of the two polarizations is defined as the polarization:

$$
P=\frac{I_{+}-I_{-}}{I_{+}+I_{-}} \text {. }
$$

To study the time dependence of the polarized intensities, we find it more convenient $[10]$ to use the total intensity

$$
I=I_{+}-I_{-}
$$

and the "spin" intensity

$$
S=I_{+}+I_{-} \text {. }
$$


The luminescence intensities of the two polarizations depend on the initial spin populations [11] of conduction electrons and valence band holes created in step (1) of Fig. 1a and spin relaxation of these carriers in two stages: during the energy and momentum relaxation of step (2) and before recombination in step (3). When the initial energy is much larger than the band gap, the electrons and holes after energy relaxation can either radiatively recombine or combine into excitons and then undergo radiative recombination. Time-resolved photoluminescence gives a measurement of the exciton formation time [5]. An alternate way to perform photoluminescence is to resonantly excite the heavy-hole excitons [12], which form a colder population than the lattice and can be scattered by phonons into a higher temperature quasi-equilibrium state before radiative recombination. It is the momentum and spin scatterings after resonant excitation which will occupy our attention here.

The first issue to raise here is the importance of studying the carrier and exciton dynamics including the spin degrees of freedom. We shall see an example later that even for experiments which involve only unpolarized light one requires for their understanding the intermediate steps involving spins.

\section{Distinction features of reduced dimensionality}

In bulk semiconductors the connection between spin dynamics and polarized light leads to the so-called the optical orientation [13]. Many of the energy and spin relaxation mechanisms in the bulk in principle apply to the quantum wells as well but in quantum wells the dependence of each mechanism on system properties differs from the bulk and, therefore, the relative importance of the various mechanisms in given ranges of temperature and carrier density is different. Four major features in quantum wells lead to distinction of the carrier and exciton dynamics from the bulk:

1. Mechanisms for radiative recombination in the quasi-two-dimensional systems are radically different from those in three-dimensional systems. Therefore, the recombination times ought to be different as well. We shall elaborate on this presently.

2. Quantum confinement of electrons leads to subband structures. An example of the confinement effect is the slow-down of the valence hole spin relaxation in the quantum well compared with the bulk, leading to a different and more satisfactory explanation of the $\mathrm{cw}$ luminescence polarization in quantum wells [11], particularly the polarization reversal effect [9].

3. The enforced close proximity of the electron-hole pair leading to a strong exciton binding in a quantum well, giving rise to the prominent role of the excitons in the optical processes in the quantum well. It is also expected to lead to a strong spin relaxation due to exciton exchange [10].

4. Molecular beam epitaxy growth of thin layers and modulation doping lead to high-mobility samples. Mechanisms which depend on motional narrowing [14], shown below to be of common occurrence, are sensitive to carrier 
momentum relaxation and should, therefore, play quite a different role for the high-mobility quantum well than for the dirty bulk semiconductor.

Radiative recombination of excitons in two-dimensional systems has been studied by a number of authors [15-18]. In a three-dimensional system, the linear coupling of photon and exciton mixes them into two branches of combined excitations, called polaritons [19], which can propagate unimpeded throughout the crystal. Decay, or recombination, can only occur in the presence of imperfections in the crystal, such as point defects, phonons, and surfaces. The recombination time is relatively long, of the order of nanoseconds. The breaking of the translational symmetry, say along the $z$-axis, leads to on the one hand a well-defined dispersion curve for the excitons versus the wave vector $k_{\|}$in the interface plane, and on the other hand to a continuum of curves for the photon given by

$$
\omega=c \sqrt{k_{\|}^{2}+k_{z}^{2}}
$$

for a whole range of values of the $z$-component of the light wave vector, with a finite and zero value of $k_{z}$ illustrated in the left-hand part of Fig. 2. For a fixed
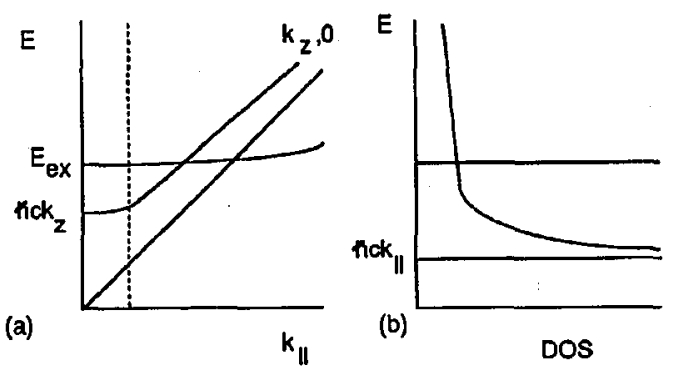

Fig. 2. (a) Dispersion curves for the two-dimensional exciton and three-dimensional photon as functions of the in-plane wave vector $k_{\|}$. (b) Densities of states of the uncoupled exciton and photon for a fixed in-plane wave vector $k_{\|}$.

$k_{\|}$, as shown by the vertical dashed line, the density of state of the photons is a continuum as shown on the right of Fig. 2, which also shows the corresponding exciton density of states as a horizontal thick line representing a $\delta$-function. The linear coupling between light and exciton can now broaden the exciton line into a peak with a finite width giving a measure of the exciton lifetime due to radiative recombination. Estimates by the authors quoted above give a lifetime of a few tens of picoseconds, i.e. between one and two order of magnitudes smaller than in three dimensions.

Yet, until recently, photoluminescence in quantum wells always leads to a radiative recombination time of the order of nanoseconds. Recent experiments with better time resolution $[6,12]$ have seen a fast initial drop in luminescence intensity but the long nanosecond tail remains. Thus, we need a theoretical framework to interpret the initial drop and to explain the long tail. 


\section{Exciton spin dynamics}

When a photon is absorbed to resonantly excite an exciton with electron and hole spins denoted by $\left(s, m_{\mathrm{h}}\right)$, the polarization of light is governed by the selection rule Eq. (2.2), i.e. light propagating along the $z$-axis with circular polarization with $m_{\mathrm{p}}$ takes on the value -1 . The $s+m_{\mathrm{h}}=-2$ excitonic states are said to be optically inactive since they cannot be created by single-photon absorption although they can be excited by two-photon processes.

Consider, for example, the lowest energy heavy-hole exciton. The $m_{\mathrm{h}}=-\frac{3}{2}$ components dominate the constituent valence hole of the exciton, so that the total exciton spin, from Eq. (2.2), is -1 or -2 . From the selection rule described above, the spin -1 excitons are optically active and the spin -2 ones are inactive. The exciton spin can be changed by flipping the spin of either the electron or the hole or by simultaneously flipping the spin of both constituents. The electron can relax its spin by the D'yakonov-Perel [20] or Elliot-Yafet [21] mechanism. Both mechanisms require substantial phase space for momentum scatterings to be effective in changing the spin. The valence hole can relax its spin through energy relaxation which changes the heavy- and light-hole mixing of the valence state [11]. The electron spin relaxation time is at least an order of magnitude longer than the hole or exciton spin relaxation time [22].

To flip the exciton spin from +1 to -1 or vice versa requires the exchange interaction [10]. The exchange interaction may be separated into a long-range part and a short-range part. The long-range part of the exchange interaction is the simple dipole interaction, of the form [23]

$$
\mu\left(2 \pi e^{2} / K\right) K_{\alpha} K_{\beta},
$$

where $K_{\alpha}$ is a component of the total momentum of the exciton. The constant $\mu$ also contains the exciton wave function at zero distance and the overlap integral in the $z$-direction. The coupling between two optically active states is analogous to a magnetic field in the $x y$-plane which will flip the exciton spin. If the center-of-mass momentum of the exciton is changed by scattering by defects or by phonons, the resulting fluctuating effective magnetic field causes spin relaxation. This is analogous to the motional narrowing effect in the nuclear spin relaxation in a metal [14]. Thus, the inverse spin relaxation time of the exciton is proportional to the time correlation of the long-range exchange matrix element or to the autocorrelation of the square of the momentum, $\left(K_{x}-\mathrm{i} K_{y}\right)^{2}$, or equivalently to the momentum relaxation time. The faster the momentum changes, the longer the spin relaxation is. The condition for the validity of this treatment is that the momentum scattering rate has to be larger than the spin precession rate. The spin relaxation by the long-range exchange requires finite center-of-mass momentum. The final wave vector acquired from the excitation light gives a negligible contribution. However, in resonant excitation of excitons, thermalization of the exciton population $[10,12]$ gives the exciton sufficient momentum for the long-range exchange to be effective. The inverse proportionality of the spin relaxation time to the momentum relaxation could also explain the sample dependence of the polarization relaxation [5].

The short-range part of the exchange interaction is of the form $\sigma-J$, where $\sigma$ and $J$ are the electron and hole spin vectors. The exchange constant is enhanced 
over the bulk value by the square of the ratio of the exciton wave functions at zero relative distance between the electron and hole times the square of the overlap subband wave functions along the $z$ direction [24]. The spin angular momentum conservation of the short-range exchange means that there is no direct matrix element which flips the exciton spin from $(-1 / 2,3 / 2)$ to $(1 / 2,-3 / 2)$ or vice versa. Only a combination of the short-range exchange with the heavy- and light-hole mixing can flip the exciton spin. For example, one can connect the heavy-hole exciton $(-1 / 2,3 / 2)$ to the light-hole exciton $(1 / 2,1 / 2)$ and then by the Luttinger Hamiltonian for the valence bands from $(1 / 2,1 / 2)$ to the heavy-hole exciton state of the opposite spin, $(1 / 2,-3 / 2)$. The latter step involves non-zero hole momentum. Thus, the momentum scattering again leads to a motional narrowing type of spin relaxation. Our estimate [10] for the GaAs/AlGaAs quantum well in Ref. [5] shows the short-range exchange contribution to be an order of magnitude less than the long-range part. With momentum scattering estimated from the width of the exciton line and the exciton temperature deduced in Ref. [5], we obtain a spin relaxation time about half that of the measured time of $50 \mathrm{ps}$. Since the exciton exchange increases in strength with decreasing well width, the spin relaxation rate, proportional to the square of the exchange interaction, is expected to increase with decreasing well width. However, the dependence is weakened somewhat by the decrease in the momentum scattering rate. Our calculated well-width dependence for the series of wells measured in Ref. [7] is in good agreement with the measured well width dependence of the polarization rate.

A way to test our theory of the spin relaxation time is to make controlled changes by external means, such as stress, magnetic or electric field along the growth axis. The effect of weak magnetic fields (i.e. without the well separated Landau levels) was given in Ref. [10]. An electric field applied along the $z$-axis (the growth direction) will polarize the exciton (increasing the separation between the electron and hole) This strongly affects the long-range part of the exchange since it depends strongly on the overlap between the electron and hole wave functions. Other possible effects caused by the introduction of the electric field, such as the increase in the impurity- and interface-related scattering processes, are neglected. The calculated increase in the spin relaxation time with the longitude electric field is in fair agreement with experiment [25]. This lends support to the role of the exchange interaction for exciton spin-flip.

\section{Time development of the electron spin populations}

The time development of the population of excitons of total spin $m_{\mathrm{p}}=s+m_{\mathrm{h}}$ is formulated as a set of rate equations $[10,26]$. The equations contain radiative recombination. We model the momentum distribution by just two sets of populations, those in the radiative recombination regime (small in-plane momenta) and those outside (large momenta). We allow for the momentum scatterings of the excitons changing their center-of-mass momenta by scatterings between these two momentum distributions. The spin-flips from -1 to -1 are due to exchange interaction via motional narrowing. The spin-flips from -1 to -2 and vice versa are given by the individual carrier spin-flips with the thermal factors governed 
by the energy difference between the spin levels, with the optically inactive states lower in energy. The recombination times and relaxations are then chosen to fit the calculated time evolution of the light emission of both polarizations to experiment $[12,25]$. The spin relaxation rates are in good order of magnitude theoretical estimates. Their dependence on the longitudinal electric field and on the well width is in good agreement with theory.

The initial fast drop of the intensities of both polarizations is due to the fast recombination time in two-dimensional systems. Part of the exciton population generated by light relaxes to the lower energy optically inactive states. Thermal excitation back to the active states as they are depleted by radiative recombination gives the long tails.

\section{Future directions}

In the moderate magnetic field regime, theory [10] shows increase in the exciton spin relaxation time but there is yet no experiment. In the high magnetic field regime, where Landau levels are important, there are experiments [27, 28] but yet no theory.

In the time regime shorter than the ones considered here, the system is dominated by the coherence effects. There are interesting many-body effects to be incorporated in the optical processes in experiments such as four-wave mixing $[29,30]$.

Another spin system can be added to the wells by doping with magnetic elements, such as has been done to $\mathrm{CdZnTe}$ quantum wells by adding Mn. This gives rise to coupling with another spin system of different spin relaxation times which can influence the exciton spins [31].

\section{Acknowledgments}

This research was supported by NSF grant No. DMR 91-17298. I acknowledge with thanks that this work was done in collaboration with M. Maialle, E. Andrada e Silva, A. Vinattieri, J. Shah, T.C. Damen, D.S. Kim, and L.N. Pfeiffer.

\section{References}

[1] Proc. Int. Conf. on the Physics of Semiconductors, back issues of Surf. Sci., J. Phys. (France), and Superlattices Microstruct. for the International Conferences on the Electronic Properties in Two Dimensional Systems, on Modulated Semiconductor Structures, and on Superlattices, Microstructures and Microdevices are good sources for the development in this field.

[2] W.H. Knox, R.L. Fork, M.C. Downer, D.A.B. Miller, D.S. Chemla, C.V. Shank, A.C. Gossard, W. Wiegmann, Phys. Rev. Lett. 54, 1306 (1985).

[3] J. Kusano, Y. Segawa, T. Aoyagi, S. Namba, H. Okamoto, Phys. Rev. B 40, 1685 (1989).

[4] T.C. Damen, J. Shah, D.Y. Oberli, D. S. Chemla, J.E. Cunningham, J.M. Kuo, Phys. Rev. B 42, 7434 (1990). 
[5] T.C. Damen, K. Leo, J. Shah, J.E. Cunningham, Appl. Phys. Lett. 58, 1902 (1991).

[6] B. Deveaud, F. Clérot, N. Roy, K. Satzke, B. Sermage, D.S. Katzer, Phys. Rev. Lett. 67, 2355 (1991).

[7] Ph. Roussignol, C. Delalande, A. Vinattieri, L. Carraresi, M. Colocci, Phys. Rev. B 45, 6965 (1992).

[8] J.M. Luttinger, Phys. Rev. 102, 1030 (1956).

[9] R.C. Miller, D.A. Kleinman, J. Lumin. 30, 520 (1985).

[10] M.Z. Maialle, E.A. de Andrada e Silva, L.J. Sham, Phys. Rev. B 47, 15776 (1993).

[11] T. Uenoyama, L.J. Sham, Phys. Rev. Lett. 64, 3070 (1990); Phys. Rev. B 42, 7114 (1990).

[12] A. Vinattieri, J. Shah, T.C. Damen, D.S. Kim, L.N. Pfeiffer, L.J. Sham, Solid State Commun. 88, 189 (1993).

[13] Optical Orientation, Eds. F. Meier, B.P. Zachachrenya, North-Holland, Amsterdam 1984.

[14] C.P. Slichter, Principles of Magnetic Resonance, Harper \& Row, New York 1963, p. 154.

[15] V.M. Agranovich, Proc. Int. Meeting on Optics of Excitons in Confined Systems, Giardini Naxos (Italy) 1991, Eds. A. Andrea, R. Del Sole, R. Girlanda, A. Quattropani, Inst. Phys. Conf. Ser. No. 123, Institute of Physics, Bristol 1992, p. 1.

[16] E. Hanamura, Phys. Rev. B 38, 1228 (1988).

[17] L.C. Andreani, F. Tassone, F. Bassani, Solid State Commun. 77, 641 (1991).

[18] D.S. Citrin, Solid State Commun. 84, 281 (1992).

[19] J.J. Hopfield, Phys. Rev. 112, 1555 (1958).

[20] M.I. D'yakonov, V.I. Perel, Sov. Phys.-JETP 33, 1053 (1971).

[21] R.J. Elliott, Phys. Rev. 96, 266 (1954); Y. Yafet, Solid State Phys. 14, 1 (1963).

[22] T.C. Damen, L. Viña, J.E. Cunningham, J. Shah, L.J. Sham, Phys. Rev. Lett. 67, 3432 (1991).

[23] G.E. Pikus, G.L. Bir, Zh. Eksp. Teor. Fiz. 60, 195 (1971) [Sov. Phys.JETP 33, $108(1971)]$.

[24] U. Rössler, S. Jorda, D.A. Broido, Solid State Commun. 73, 209 (1990).

[25] A. Vinattieri, J. Shah, T.C. Damen, K.W. Goossen, L.N. Pfeiffer, M.Z. Maialle, L.J. Sham, Appl. Phys. Lett. 63, 3164 (1993).

[26] M.Z. Maialle, L.J. Sham, Surf. Sci. 305, 256 (1994).

[27] J.B. Stark, W.H. Knox, D.S. Chemla, Phys. Rev. B 46, 7919 (1992).

[28] M. Potemski, J.C. Maan, A. Fasolino, K. Ploog, G. Weimann, Phys. Rev. Lett. 63, 2409 (1989).

[29] S.T. Cundiff, H. Wang, D.G. Steel, Phys. Rev. B 46, 7248 (1992).

[30] D.S. Kim, J. Shah, T.C. Damen, W. Schäfer, F. Janhnke, S. Schnitt-Rink, K. Köhler, Phys. Rev. Lelt. 69, 2725 (1992).

[31] J.J. Baumberg, D.D. Awschalom, N. Samarth, H. Luo, J.K. Furdyna, Phys. Rev. Lett. 72, 717 (1994). 